P-ISSN: 2664-3685

E-ISSN: 2664-3693

www.paediatricjournal.com IJPG 2019; 2(2): 101-104

Received: 16-05-2019

Accepted: 18-06-2019

Rini R Naik

Senior Resident, Department of Obstetrics and Gynaecology, Goa Medical College,

Bambolim, Goa, India

\section{Guruprasad Pednekar}

Professor and Head,

Department of Obstetrics and

Gynaecology, Goa Medical

College, Bambolim, Goa, India

Jagadish Cacodcar

Professor and Head,

Department of Preventive and

Social medicine, Goa Medical

College, Bambolim, Goa, India

\section{Analysis of outcomes in neonates of mothers with gestational diabetes mellitus at a tertiary care hospital in Goa}

\author{
Rini R Naik, Guruprasad Pednekar and Jagadish Cacodcar
}

DOI: https://doi.org/10.33545/26643685.2019.v2.i2b.42

\begin{abstract}
Background: Diabetes is a common metabolic disorder complicating pregnancy in India. Babies born to diabetic mothers are at increased risk of neonatal complications as well as long term complications with potential of developing type $2 \mathrm{DM}$ in future.

Methods: A large prospective study was conducted at a tertiary care hospital in Goa for a period of 18 months (2014-2016) to study the outcomes among neonates born to mothers with Gestational Diabetes Mellitus (GDM)

Results: Clinical outcomes of neonates born to 424 mothers with GDM were studied and compared with 424 neonates born to mothers without GDM during the same time period. The mean birth weight of neonates born to GDM mothers was $3.17 \mathrm{~kg}$. Macrosomia was found among $1.2 \%$ neonates born to GDM mothers. There was no significant difference in Apgar scores at 5 minutes of birth in GDM and non-GDM groups. The rate of admissions to Neonatal Care Unit was significantly higher $(32.1 \%)$ in neonates born to GDM mother as compared to non-GDM group. Incidence of neonatal complications like respiratory distress syndrome $(4.2 \%)$ and hypoglycaemia $(11 \%)$ was significantly higher in neonates born to GDM mothers. There was a significant increase noted in neonatal mortality (3.3\%) in the GDM group.

Conclusion: Numerous complications are reported among neonates born to mothers with GDM. Hence these mothers with GDM should be delivered in tertiary care centre or district hospitals where fully staffed and well equipped neonatal intensive care units are available for management of these complications.
\end{abstract}

Keywords: Macrosomia, hypoglycemia, NICU admissions, neonatal morbidity

\section{Introduction}

Infants born to mothers with GDM are more prone to numerous complications which may be fetal, neonatal and even long term. Such infants are at increased risk of fetal and neonatal complications like congenital anomalies, stillbirth, macrosomia, shoulder dystocia, birth injuries, birth asphyxia, respiratory distress syndrome (RDS), hypoglycaemia, hyperbillirubinemia, hypocalcemia, etc. ${ }^{[1]}$. Macrosomia is defined as fetal birth weight above the $90^{\text {th }}$ percentile for gestational age or $>4 \mathrm{~kg}$. Macrosomia is found in $15-45 \%$ of diabetic pregnancies, which is 3 times more compared to those with normal glucose levels. Pederson's hypothesis states that maternal hyperglycemia leads to fetal hyperinsulinemia and increased glucose utilization and hence increases adipose tissue of the fetus. In GDM, as the maternal blood glucose levels increase it crosses the placenta but the maternal insulin or exogenously administered insulin doesn't cross the placenta. Thus in the second trimester, the fetal pancreas secrete insulin autonomously secondary to hyperglycemia irrespective of glucose stimulation. Thus a combined effect of hyperinsulinemia and hyperglycemia leads to an increase in fat and protein stores leading to macrosomia ${ }^{[2]}$. Complications of macrosomia in the mother include- prolonged labour, increased incidence of operative delivery and perineal tears. In the fetus, complications include - shoulder dystocia, erb's palsy and long term complications like, neurological damage and metabolic syndrome.

Gestational diabetes is associated with a significant risk of holoprosencephaly, upper/lower spine or rib defects, and renal and urinary system anomalies. ${ }^{[3]}$ Martínez-Frías et al. suggested that holoprosencephaly is significantly associated with gestational diabetes while neural tube defects are not ${ }^{[3]}$.

Insulin interferes with substrate availability for surfactant biosynthesis. Insulin also interferes with the normal timing of glucocorticoid induced pulmonary maturation in the fetus. However the risk of RDS in GDM babies at term is no more than the risk among non-GDM babies ${ }^{[4]}$.
Corresponding Author: Rini R Naik

Senior Resident, Department of Obstetrics and Gynaecology, Goa Medical College,

Bambolim, Goa, India 
Neonatal hypoglycaemia is defined as blood glucose level < 35 to $40 \mathrm{mg} / \mathrm{dl}$ during the first 12 hours of life ${ }^{[5]}$. It is commonly seen in macrosomic babies. It results from a rapid fall in plasma glucose concentrations following clamping of the umbilical cord while levels of insulin are still high in the newborn.

Hyperbilirubinemia is more common among neonates born to GDM mothers than non GDM mothers, but is still fairly infrequent. In the HAPO study, hyperbilirubinemia was defined as treatment with phototherapy after birth, or at least one laboratory report of a bilirubin level $20 \mathrm{mg} / \mathrm{dL}$, or readmission to the hospital for hyperbilirubinemia ${ }^{[6]}$. Maternal hyperglycemia and the subsequent induction of fetal hyperinsulinemia and reduced oxygenation are hypothesized to lead to increased fetal oxygen uptake, fetal erythropoiesis, and subsequent hyperbilirubinemia.

Hypocalcemia (serum calcium $<7 \mathrm{mg} / \mathrm{dl}$ ) is also reported in more frequency among neonates born to GDM mothers ${ }^{[7]}$.

Adequate management of GDM and good glycemic control can improve perinatal outcomes in GDM. Due to increased risk of perinatal morbidity and mortality associated with GDM, neonates born to GDM mothers should be intensively monitored. The present study was conducted to identify and analyse the outcomes in neonates born to mothers with GDM to generate evidence and improve perinatal outcomes.

\section{Materials and Methods}

The current large prospective hospital based longitudinal study was conducted in the Department of Obstetrics and Gynaecology at a tertiary care hospital in Goa for a period of 18 months among neonates born to mothers with GDM between November 2014 and April 2016. The approval of the Institutional Ethical Committee was obtained prior to commencement of the study. Informed consent was obtained from all the study participants.

All the pregnant mothers delivering in our hospital during the above mentioned time period and gave consent were included in the study. All patients with overt diabetes mellitus, major chronic diseases like cardiac failure, hepatorenal failure and tuberculosis and with multiple gestations were excluded from the study. All the antenatal mothers included in the study were universally screened for GDM using oral glucose challenge test (OGCT) with 50g glucose at the first visit. If the plasma glucose level measured at the end of 1 hour was more than $140 \mathrm{mg} / \mathrm{dl}$, then such mothers were subjected to the diagnostic test - Oral Glucose tolerance test (OGTT) with $100 \mathrm{~g}$ glucose. If the OGCT value was normal then the screening test was repeated at 24 - 28 weeks of gestation. Antenatal mothers with any high risk factors were directly subjected to the diagnostic test at the first antenatal visit. Once GDM was diagnosed, the patients were advised medical nutritional therapy (split meals), exercises and were followed up every 15 days for evaluation of glucose control. If FBSL was $>95 \mathrm{mg} / \mathrm{dl}$ and postmeal RBSL was > $120 \mathrm{mg} / \mathrm{dl}$, insulin therapy was initiated.

All pregnant mothers with GDM that did not go in spontaneous labour by 38 weeks of gestation were induced. Their neonatal outcomes were compared to the equal number of neonates of randomly chosen antenatal mothers without GDM who had delivered at our hospital during the same time period. Neonatal complications like macrosomia, RDS, neonatal hypoglycaemia and neonatal hyperbilirubinemia were studied amongst the neonates born to GDM mothers. Apgar scores at 1 and 5 minutes of birth, weight at birth, rate of NICU admissions and number of neonatal deaths were recorded. A stay in NICU of more than 24 hours indicated neonatal morbidity. The results were analyzed using SPSS (version 22). Chi square test was applied as a test of significance and $\mathrm{p}$ value $<0.05$ was considered statistically significant.

\section{Results}

The clinical outcomes of neonates born to 424 mothers with GDM were studied and compared with 424 neonates born to mothers without GDM during the same time period. In the present study, approximately one third of the mothers with GDM delivered between 38.1 - 39 weeks of gestation $(34.7 \%)$. A significantly higher percentage of neonates born to mothers with GDM (17\%) were preterm as compared to $9.9 \%$ in the non-GDM group $(P 0.0035)$. The mean birth weight of babies born to mothers with GDM was $3.17 \mathrm{~kg}$, whereas the mean birth weight in the non-GDM group it was $2.63 \mathrm{~kg}$. Table 1 shows the birth weight distribution in neonates born to GDM and Non GDM mothers.

Table 1: Birth weight distribution in neonates born to GDM and non- GDM mothers

\begin{tabular}{|c|c|c|c|c|}
\hline \multirow{2}{*}{ Weight (kg) } & \multicolumn{2}{|c|}{ GDM group } & \multicolumn{2}{c|}{ non-GDM group } \\
\cline { 2 - 5 } & No. of patients & Percentage (\%) & No. of patients & Percentage (\%) \\
\hline$<2$ & 15 & 3.5 & 20 & 4.7 \\
\hline $2-2.5$ & 18 & 4.2 & 148 & 35 \\
\hline $2.6-3$ & 129 & 30.4 & 205 & 48.3 \\
\hline $3-3.5$ & 144 & 34 & 42 & 9.9 \\
\hline $3.5-4$ & 113 & 26.7 & 7 & 1.6 \\
\hline$>4$ & 5 & 1.2 & 2 & 0.5 \\
\hline
\end{tabular}

Macrosomia was diagnosed among $1.2 \%$ of neonates born to GDM mothers as compared to $0.5 \%$ in the non GDM group; however this association was not statistically significant $(P 0.4478)$.

Only 4 neonates $(0.9 \%)$ in the GDM group had Apgar score of less than 6 at 5 minutes of birth as opposed to $3(0.7 \%)$ in the non-GDM group. There was no significant association between GDM and Apgar score at birth ( $P$ 1.0000).
Table 2: Incidence of RDS in neonates born to GDM mothers versus non- GDM mothers

\begin{tabular}{|c|c|c|c|c|c|}
\hline \multicolumn{3}{|c|}{ GDM group } & \multicolumn{3}{c|}{ non - GDM group } \\
\hline RDS & $\begin{array}{c}\text { No. of } \\
\text { patients }\end{array}$ & $\begin{array}{c}\text { Percentage } \\
(\boldsymbol{\%})\end{array}$ & RDS & $\begin{array}{c}\text { No. of } \\
\text { patients }\end{array}$ & $\begin{array}{c}\text { Percentage } \\
(\%)\end{array}$ \\
\hline Yes & 18 & 4.2 & Yes & 7 & 1.7 \\
\hline No & 406 & 95.7 & No & 417 & 98.3 \\
\hline
\end{tabular}


As seen in Table 2 above; among the neonates born to GDM mothers, $18(4.2 \%)$ had respiratory distress syndrome (RDS) which was significantly higher than $7(1.7 \%)$ neonates in the non GDM group ( $P$ 0.0423).

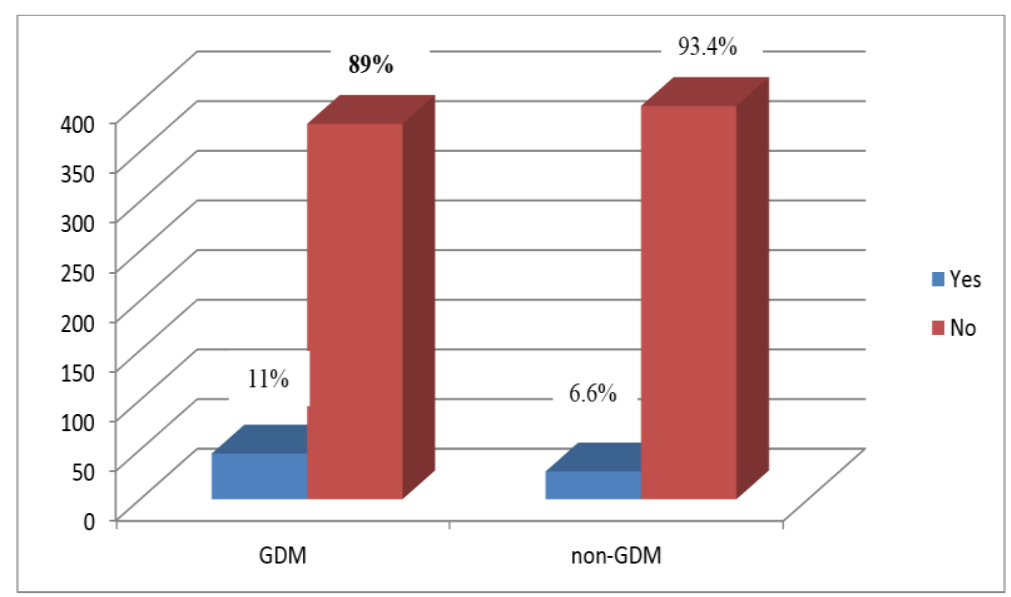

Fig 1: Comparison of percentage of neonates developing hypoglycaemia in GDM versus non-GDM groups.

Figure 1 shows that a higher incidence of neonatal hypoglycaemia was noted in the GDM group as compared to non-GDM group $(P \quad 0.0386)$. Neonatal hyperbilirubinemia was detected in $35 \%$ of neonates in the GDM group as opposed to $38.3 \%$ in the non-GDM group. There was no significant association between GDM and neonatal hyperbilirubinemia $(P$ 0.3539). $13 \%$ of neonates born to mothers with GDM mothers had developed neonatal sepsis as compared to $9 \%$ of the neonates in the non-GDM group. However this finding was not statistically significant (P 0.0787).

Table 3: NICU admissions among neonates of GDM and non-GDM mothers

\begin{tabular}{|c|c|c|c|c|c|}
\hline \multicolumn{3}{|c|}{ GDM group } & \multicolumn{3}{c|}{ non - GDM group } \\
\hline NICU Admissions & No. of patients & Percentage (\%) & NICU Admissions & No. of patients & Percentage (\%) \\
\hline Yes & 136 & 32.1 & Yes & 101 & 23.8 \\
\hline No & 288 & 67.9 & No & 323 & 76.2 \\
\hline
\end{tabular}

As seen in Table 3 NICU admissions were more common in neonates born to mothers in GDM group than among neonates belonging to non-GDM group. This result was statistically significant $(P 0.0093)$

Table 4: Percentage of neonatal deaths among GDM and non-GDM groups

\begin{tabular}{|c|c|c|c|c|c|}
\hline \multicolumn{3}{|c|}{ GDM group } & \multicolumn{3}{c|}{ non - GDM group } \\
\hline Neonatal Deaths & No. of patients & Percentage (\%) & Neonatal Deaths & No. of patients & Percentage (\%) \\
\hline Yes & 14 & 3.3 & Yes & 4 & 0.9 \\
\hline No & 410 & 96.7 & No & 420 & 99.1 \\
\hline
\end{tabular}

As seen in Table 4, it is inferred that GDM was associated with significant neonatal mortality $(P 0.0320)$.

\section{Discussion}

Diabetes is the most common endocrine disorder in pregnancy which is known to have adverse maternal and perinatal outcomes. The onset, duration and severity of maternal hyperglycemia and glycemic control during pregnancy establish the neonatal outcome.

In the present study 424 neonates born to mothers with GDM were examined and followed up and their outcomes were compared to equal number of randomly selected neonates born to mothers without GDM.

The mean birth weight in GDM was found to be $3.17 \mathrm{~kg}$ in the present study. Jayshree et al. ${ }^{[8]}$ found a mean birth weight of $2.86 \mathrm{~kg}$ in the GDM group. Ameya Dudhwadkar et al. ${ }^{[9]}$ found that $40 \%$ of babies born to GDM mothers had birth weight of $>3.5 \mathrm{~kg}$. The present study showed that the percentage of neonates born to GDM mothers developing macrosomia was $1.2 \%$ which was much lower as compared to other studies like Jayshree et al. $(2.3 \%)^{[8]}$, Rafiq W. et al. $(39.8 \%)^{[10]}$, Dharmavijaya MN et al. (3.4\%) ${ }^{[11]}$, Mohan
Makwana et al. (18.42\%) ${ }^{[12]}$, Amidha et al. 14\%) ${ }^{[13]}$ and Anjum et al. (15\%) ${ }^{[14]}$. This is because of early detection, systematic antenatal surveillance and good glycemic control achieved in our hospital setting. The present study showed a higher incidence of prematurity in neonates born to mothers with GDM (17\%). However studies done by Jayshree et al. ${ }^{[8]}$, Ameya Dudhwadkar et al. ${ }^{\text {[9] }}$ and Amidha et al. [13] showed no significant association with prematurity and GDM. This difference was noted because in the present study, a higher incidence of genitourinary infections was observed in mothers with GDM which predisposed them to preterm labour. In the present study, only $0.9 \%$ of babies born to GDM mothers had five minute Apgar score of 6 or less which did not have any significant association with GDM. Rafiq W. et al. ${ }^{[10]}$ have reported a significantly higher percentage $(10.41 \%)$ and Mohan Makwana et al. ${ }^{[12]}$ observed that $18.42 \%$ of neonates born to GDM mother had low Apgar score at 5 minutes of birth. Thus in our study better Apgar scores were observed due to less labour complications such as shoulder dystocia, as the incidence of macrosomia was significantly less in our study as compared to the others. 
RDS was observed in a significantly higher percentage i.e $4.2 \%$ of neonates born to GDM mothers. Similarly in other studies Ameya Dudhwadkar et al. ${ }^{[9]}$, Rafiq W. et al. ${ }^{[10]}$, Mohan Makwana et al. ${ }^{[12]}$ and Anjum et al. ${ }^{[14]}$ reported significant increase in the incidence of RDS in neonates born to GDM mothers. The higher percentage of RDS was attributed to an increased incidence of prematurity associated with GDM.

Neonatal hypoglycemia was observed in a significantly higher percentage in GDM group in our study $(11 \%)$ which was comparable with findings of other studies. $[9,10,11,12,13,14]$ Our study results showed no significant increase in the incidence of hyperbilirubinemia in neonates born to GDM mothers. However several other studies reported a significant rise in the incidence of neonatal hyperbilirubinemia in babies born to GDM mothers. [9, $10,11,12,13,14]$ This difference was observed because there was a higher percentage of neonatal hyperbilirubinemia in the non-GDM group as compared to GDM group in our study due to multiple factors in the mothers in non-GDM group such as Rh negative pregnancy, ABO incompatibility, small for gestational age babies, prematurity, breast feeding issues, etc.

In the present study, $13 \%$ of neonates born to GDM mothers had neonatal sepsis which was higher than that observed by Jayshree et al. $(2.3 \%)^{[8]}$, Ameya Dudhwadkar et al. (4\%) ${ }^{[9]}$ and Dharmavijaya $\mathrm{MN}$ et al. $(7.5 \%)^{[11]}$. Increased incidence of genitourinary infections, preterm labour and prelabour rupture of membranes in our study probably contributed towards the increased incidence of neonatal sepsis in our study.

We observed in our study that there was a significantly high rate of NICU admission (32.1\%) in neonates born to GDM mothers. This was attributed to increased incidence of prematurity and neonatal complications like hypoglycemia, RDS and neonatal sepsis. Our study finding is similar to other studies like Jayshree et al. ${ }^{[8]}$ (27.6\%) and Mohan Makwana et al. ${ }^{[12]}(31.58 \%)$.

The neonatal mortality in GDM group was significantly high in our study $(3.3 \%)$. Jayshree et al. ${ }^{[8]}$ found the neonatal mortality to be $3.5 \%$ in GDM group. Dharmavijaya $\mathrm{MN}$ et al. ${ }^{[11]}$ reported neonatal mortality of $1.4 \%$ in GDM group while Amidha et al. ${ }^{[13]}$ reported it to be $6 \%$. However in all these studies the neonatal mortality was not significantly increased in neonates born to mothers with GDM. Increased incidence of prematurity and RDS contributed to this increased neonatal mortality rate in our study.

\section{Conclusions}

We conclude that GDM is associated with numerous adverse neonatal outcomes and increases the risk of perinatal morbidity and mortality. GDM is on the rise in the South East Asian population, especially India. Hence appropriate measures need to be implemented to prevent, detect and treat GDM early in pregnancy. Universal screening for GDM must be made mandatory. Preganant women with GDM should be delivered in tertiary care centres or centres with well equipped and fully staffed neonatal units in order to improve the neonatal morbidity associated with GDM.

\section{References}

1. Rosca Daniela. Fetal and neonatal complications of diabetic pregnancy. The Moldovan Medical Journal 2017; 60(4):49-55.

2. Pederson J. The pregnant woman and her newborn. 2nd edition Baltimore Williams Wilkins, 1997.

3. Martínez-Frías ML, Bermejo E, Rodríguez-Pinilla E, Prieto L, Frías JL. Epidemiological analysis of outcomes of pregnancy in gestational diabetic mothers. Am J Med Genet. 1998; 78:140-5.

4. Berkowitz K, Reyes C et al. Comparison of fetal lung maturation in well dated diabetic and non diabetic pregnancies. AM J Obstet Gynecol. 1996; 174:373.

5. Langer et al. complications in newborns of Diabetic Mothers. Obstet Gynaecol N Am. 2007; 34:241-243.

6. HAPO. Study Cooperative Research Group. Hyperglycemia and adverse pregnancy outcomes. N Engl J Med. 2008; 358(19):1991-2002.

7. Cruikshank DP, Pitkin RN et al. Altered maternal calcium homeostasis in diabetes. J Clinical endocrinol Metab. 1980; 50:264.

8. Jayshree Mulik et al. Study of Maternal and Perinatal outcome in Pregnancies Complicated by Gestational Diabetes Mellitus. Indian journal of Applied Research. 2016; 6(7):64-66.

9. Dudhwadkar AR, Fonseca MN. Maternal and fetal outcome in gestational diabetes mellitus. Int J Reprod Contracept Obstet Gynecol. 2016; 5:3317-21.

10. Rafiq W, Hussain SQ, Jan M, Najar BA. Clinical and metabolic profile of neonates of diabetic mothers. Int $\mathrm{J}$ Contemp Pediatr. 2015; 2:114-8.

11. Dharmavijaya MN, Chandra Mouli, Jyoti Kamda. Analysis of gestational diabetes mellitus from a tertiary care hospital. Indian Journal of Obstetrics and Gynecology Research. 2017; 4(1):17-20.

12. Makwana M, Bhimwal RK, Ram C, Mathur SL, Lal K, Mourya H. Gestational diabetes mellitus with its maternal and foetal outcome-a clinical study. Int J Adv Med. 2017; 4:919-25.

13. Amidha Shukla, Shankar Burute, Aarti Meena. Maternal and fetal outcome in gestational diabetes - A retrospective study. International Journal of Applied Research. 2017; 3(9):305-309.

14. Anjum SK, Yashodha HT. A study of neonatal outcome in infants born to diabetic mothers at a tertiary care hospital. Int J Contemp Pediatr. 2018; 5:489-92. 